\title{
Initial spin periods of neutron stars in supernova remnants
}

\author{
S.B. Popov ${ }^{1}$ \\ Sternberg Astronomical Institute, Lomonosov Moscow State University, Russia \\ sergepolar@gmail.com \\ and \\ R. Turolla \\ Department of Physics and Astronomy, University of Padova, Italy \\ Mullard Space Science Laboratory, University College London, UK
}

Received __; accepted _ _

Not to appear in Nonlearned J., 45.

\footnotetext{
${ }^{1}$ Visiting Scientist, University of Padova
} 


\begin{abstract}
We present estimates of initial spin periods, $P_{0}$, for radio pulsars associated with supernova remnants. By using the published data on 30 objects, we were able to derive a reliable estimate for the initial spin period, assuming standard magneto-dipole spin-down (braking index $n=3$ ), in many cases. Our set of estimates is still not sufficient to infer the exact shape of the initial period distribution. However, we show that a gaussian distribution with mean and deviation $\sim 0.1 \mathrm{~s}$ is consistent with our results, while flat, wide distributions and very narrow ones are disfavored.
\end{abstract}

Subject headings: neutron stars 


\section{Introduction}

Studies of neutron star (NS) properties and evolution is a fruitful field of research uniting astrophysics and fundamental physics (see a general review and references, for example, in the introductory part of the book Haensel et al. 2007 and a shorter review in Lattimer and Prakash 2004). In the map of this research area quantum chromodynamics is linked to observations of cooling NSs; general relativity with pulse profiles of X-ray sources; strong field electrodynamics with astrophysics of radiopulsars and magnetars, and so on. Despite significant progress, some parts of this picture are still obscure. One of them is related to NSs initial parameters. The knowledge of initial properties of compact objects is of crucial importance not only for understanding their evolution and modelling their observational appearance, but also because the parameters of newborn NSs carry the imprint of the still poorly understood supernova (SN) explosion mechanism.

For a NS the main initial parameters are the mass $(M)$, dipolar external magnetic field $(B)$, spin period $(P)$ and spatial velocity $(v)$. In addition, the angle between the spin and magnetic axis, the initial configuration of the crustal magnetic field (including a toroidal component), and other parameters are important for the thermal and/or magneto-rotational evolution and for fixing the different observational manifestations of NSs.

There is strong, ongoing effort by many research groups in the attempt to advance our knowledge of NSs initial parameter distributions. This can be done by different techniques. Direct observations, unfortunately, are impossible, but observational data can be used together with assumptions about the NS evolution to estimate a posteriori the initial parameters for individual objects. Another approach is related to population synthesis (Popov and Prokhorov 2007). In this case an evolutionary scenario is applied to follow numerically a large sample of sources, selection effects are taken into account, and results are confronted with observational distributions. Finally, initial parameters can be calculated 
in the framework of some theoretical model (for example, models of SN explosion).

Population synthesis modelling was used to study the initial properties of radiopulsars (PSRs) by Faucher-Giguère and Kaspi (2006), who considered several possible initial distributions of NS parameters in the case of standard PSRs. Another approach was used in Popov et al. (2010), where in the framework of a decaying magnetic field, several types of isolated NSs were studied, with a focus on the properties of the initial magnetic field. A direct numerical model is used, for example, to derive initial mass distribution of compact objects (e.g. Fryer et al. 2011). Another example is related to the calculation of the kick (recoil) velocity of newborn compact objects (Scheck et al. 2006).

In this note we address the problem of determining the initial spin period $P_{0}$ of NSs. This is done by using data on the "true age" of NSs, as obtained by observations of associated SN remnants (SNRs) and applying a standard model of spin evolution. In the next section we present our sample and results. Then we provide a discussion of several issues related to our study, and, finally, conclude.

\section{Sample and initial periods}

We collected a sample of 30 NSs with well-measured $P$ and $\dot{P}$, associated with SNRs or/and pulsar wind nebulae (see Table 1 for a complete list). For all of them there are estimates of SNR ages based on different assumptions (in the case of the Crab nebula and, probably, G11.2-0.3 historical ages can be used, see Green and Stephenson 2003). Period and period derivatives are taken from the ATNF catalogue (Manchester et al. 2005)1. 1 .

In 6 cases the independently estimated SNR age, $\tau_{\mathrm{SNR}}$, is consistent with the spin-down

\footnotetext{
${ }^{1}$ The on-line catalogue is available at http://www.atnf.csiro.au/people/pulsar/psrcat/
} 
age, $\tau_{\mathrm{sd}}=P / 2 \dot{P}$, calculated for zero initial spin periods and braking index $n=3\left(n \equiv \nu \ddot{\nu} / \dot{\nu}^{2}\right.$, where $\nu=1 / P$ is the spin frequency), and so no estimate of $P_{0}$ is possible in the standard picture, except that it has to be $P_{0} \ll P$. Note, that this does not formally mean that $P_{0} \simeq 0$. The initial period for these sources can range from a few ms up to tens of ms (as in, for example, PSR J1119-6127 and PSR J1846-0258). In plotting the estimated initial spin period for these sources (Fig. 1) we assume $P_{0}<0.1 P$.

In three cases only upper or lower limits on a SNR age are available. For 15 SNRs age ranges are reported in the literature. For most of them it is possible to obtain a range of initial spin periods and for some the resulting ranges for $P_{0}$ are narrow. In several cases $\tau_{\text {sd }}$ is in between the upper and lower limit of $\tau_{\mathrm{SNR}}$. Correspondingly, only an upper limit on $P_{0}$ can be derived. Finally, in 6 cases the SNR ages are relatively well determined, and in one case the uncertainty is tiny in comparison with the spin-down age of the pulsar. This allows us to obtain firm estimates of $P_{0}$ for a given braking index.

Initial spin periods are estimated from the usual expression

$$
P_{0}=\left[P^{n-1}-(n-1) \dot{P} P^{n-2} \tau_{\mathrm{SNR}}\right]^{1 /(n-1)}
$$

where $P$ and $\dot{P}$ are the present values of the spin period and period derivative, and $n$ is the braking index. For $n=3$ the equation reduces to the usual magneto-dipole formula with $B \propto \sqrt{P \dot{P}}$. These are the values presented in Table 2 and Fig. 1. The field is assumed to be constant during the lifetime of a NS, as well as the angle between spin and magnetic axes. This assumption is well justified, as all sources are young and do not possess strong magnetic fields. So, it is very unlikely that field decay could play a major role in their evolution. Our estimates are in good correspondence with those provided by Migliazzo et al. (2002). However, our list is significantly broader, although it is based on less detailed observational data. 


\section{1. $\quad$ Notes on individual objects}

N157B. The source is situated in the Large Magellanic Cloud. The pulsar was discovered by Marshall et al. (1998) and the age of the associated Crab-like SNR is estimated in Wang and Gotthelf (1998) as 5000 yrs basing on X-ray data (ROSAT and ASCA). Uncertainties in the age are not reported. The SNR age is in good correspondence with the characteristic age of the pulsar.

G292.2-0.5. Both for free expansion and Sedov stage Pivovaroff et al. (2001) conclude that the age of the nebula can be in correspondence with the radio pulsar characteristic age.

G0.9+0.1. This is a composite SNR. Age estimates reported by Aharonian and et al. (2005) and Porquet et al. (2003) are not precise, a few thousand years. This is compatible with the characteristic age of the pulsar.

G359.23-0.82. This is a pulsar wind nebula. There are no reliable age estimates for this object which can be used for deriving the radio pulsar initial spin period. Formally, the age of the nebula is consistent with the pulsar characteristic age.

Kes 75. Basing on the detailed analysis of the distance towards this object Leahy and Tian (2008) conclude that the age of the SNR is in correspondence with the radio pulsar age.

G54.1+0.3. This is a Crab-like nebula. Camilo et al. (2002a) reported that no independent reliable age estimate exists for this source. Roughly, the age of the nebula is consistent with the pulsar spin-down age.

CTA 1. Halpern et al. (2004) report an age of 13,000 yrs for the SNR age, referring

to Slane et al. (2004). This value corresponds to a distance to the remnant of $1.4 \mathrm{kpc}$. The uncertainty, according to Slane et al. (2004), is $\pm 0.3 \mathrm{kpc}$, which translates in to \pm 2800 yrs for the uncertainty in the age. 
3C 58. In a recent paper Slane et al. (2008) report the value of 5400 yrs referring to earlier studies. Bietenholz (2006) gives 7000 yrs with a $3 \sigma$ lower limit of 4300 years. Rudie and Fesen (2007) suggest a value of "about few thousand years". Finally, Chevalier (2005) has $2400 \pm 500$ yrs. In our study we use the interval 4300-7000 yrs.

S147. Old age estimates (see, for example, Kundu et al. 1980) suggested a SNR age of $\sim 80-200$ kyrs. In a recent paper $\mathrm{Ng}$ et al. (2007) gave arguments in favour of shorter age, $\sim 40$ kyrs. The kinematic age of the pulsar is also close to this value.

0540-693. Several estimates are available for this source. Manchester et al. (1993) give $760 \pm 100$ yrs referring to older studies, in particular to Reynolds (1985) and Kirshner et al. (1989). Reynolds (1985) presents an estimate of 800-1100 years. Kirshner et al. (1989) give $762 \pm 100$ yrs. Williams et al. (2008) and Park et al. (2010) provide wide lists of references and age estimates for this source. For our study we use an interval 660-1100 yrs.

Monogem Ring. Thorsett et al. (2003) give a single value, 86,000 yrs, as the age estimate for this object. They refer to Plucinsky et al. (1996), where this value corresponds to a distance of 300 pc. However, possible distances range from 100 to 1300 pc. This can translates in to a 29,000-371,000 yrs interval for the age estimate. These authors state that small and large distances are not very probable, but $d=600 \mathrm{pc}$ is certainly as good as 300 pc. This distance estimate $(600 \mathrm{pc})$ corresponds to the age 170,000 yrs. So, we use the interval 86-170 kyrs.

Puppis A. Gotthelf and Halpern (2009) refer to Winkler et al. (1988), where an estimate of $3700 \pm 300 \mathrm{yrs}$ is given. We use in our analysis the range 3400-4100 yrs reported by Gotthelf and Halpern (2009).

Vela. For the Vela pulsar the upper limit for the age is taken from Aschenbach et al. (1995). These authors used $d=500 \mathrm{pc}$, a value which is currently revised towards lower 
values which result in a smaller upper limit for the age. The lower limit for this SNR is not very certain, and we use the value 11 kyr. Slightly smaller values are possible, but this would not change our conclusions about the initial period significantly. Strictly speaking, the age of the Vela remnant can be considered just in correspondence with the characteristic age of the pulsar. Still, we provide a full range for age estimates reported in the literature, and use the source in our analysis.

G292.0+1.8. Gonzalez and Safi-Harb (2003) give the range 2400-2850 yrs, which we use for our estimates.

G320.4-1.2. The estimates of the SNR age given by Yatsu et al. (2005) are based on standard assumptions about the SN explosion energy and ISM density. So, the age estimate is rather uncertain, probably the nebula's age is in correspondence with the pulsar age. We do not provide any estimated of $P_{0}$ for this object.

G7.5-1.7. The estimate of $10^{4}-10^{5}$ yrs given by Roberts and Brogan (2008) is based on numerical estimates for SNRs of this type, and as such is quite rough. The authors also suggest 50,000 yrs as a representative value. We use the full range above.

G12.8-0.0. We use the range of ages from Brogan et al. (2005). In Dean and Hill (2008) the properties of the radio pulsar are discussed in details, and arguments in favour of significant initial period (i.e., compatible with the present day one) are provided.

G21.5-0.9 Safi-Harb et al. (2001) provide a list of references for age estimates of the SNR. We use the full reported range which is rather wide.

W44. Harrus et al. (1997) present two possibilities. The first one gives a SNR age of 5600-7500 yrs with a central value 6500 yrs, the second one 19000-25000 yrs. In a recent paper Abdo and et al. (2010) used an estimate $\sim 20000$ yrs. For our estimates we use the range 6.5-20 kyrs. 
G65.1+0.6. The large uncertainty in the age of the SNR is not influential on our results, as anyway the values are much smaller than the value of the characteristic age of the pulsar.

CTB80. There is only an age estimate for the pulsar wind nebula. We consider this as a lower limit for the actual age of the pulsar, so only an upper limit for $P_{0}$ can be obtained.

G308.8-0.1. Caswell et al. (1992) report just an upper limit for $\tau_{\text {SNR }}$ because the value of the ISM density is uncertain (in particular it can be lower). This estimate is larger than $\tau_{\text {sd }}$, and no conclusion about $P_{0}$ can be reached.

G106.6+2.9. Kothes et al. (2006) obtained an estimate for the pulsar wind nebula age. We use this as a lower limit for the age of the pulsar.

Crab. The age of this SNR is well-known as it is related to a historical SN.

G296.5+10.0. Vasisht et al. (1997) give an estimate of $\sim 10^{4}$ yrs for an explosion energy $E=210^{51} \mathrm{erg}$ and an ISM density of $0.2 \mathrm{~cm}^{-3}$. They refer to Kellett et al. (1987) who provide an estimate 20,000 yrs for $E=1.510^{51} \mathrm{erg}$ and ISM density of $0.26 \mathrm{~cm}^{-3}$. Both values are extremely small in comparison with the spin-down age of the pulsar.

G315.9-0.0. Camilo et al. (2009) calculate an age estimate basing on the assumption that the remnant is at the Sedov stage of expansion. Correspondingly, uncertainties are related to uncertainties in distance, ISM density, and SN explosion energy, which are however not discussed.

G11.2-0.3. The age of this SNR is considered to be well established as it is a historical event (Torii et al. 1999). In addition, Sedov phase estimates are consistent with the historical interpretation (Vasisht et al. 1996).

Kes 79. Uncertainties in the age for this SNR are related to uncertainties in its 
distance, which is of the order of $\pm 1 \mathrm{kpc}$. Taking into account that the former are at the $\sim 15-20 \%$ level and that the SNR age is orders of magnitude smaller than the characteristic age of the pulsar, we neglect the uncertainty.

G78.2+2.1. Exact uncertainty in the age is not reported by Uchiyama et al. (2002), but it is anyway small and does not influence our results.

G114.3+0.3. Exact uncertainty in the age is not reported by Yar-Uyaniker et al. (2004), but it is anyway small and does not influence our results. 
Table 1. Sample of PSRs associated with SNRs

\begin{tabular}{|c|c|c|c|c|}
\hline PSR & SNR & $\tau_{S N R} / 10^{3} \mathrm{yrs}$ & $\tau_{s d} / 10^{3} \mathrm{yrs}$ & Ref. \\
\hline J0537-6910 & N157B & as the PSR & 4.9 & Wang and Gotthelf (1998) \\
\hline J1119-6127 & G292.2-0.5 & as the PSR & 1.6 & Pivovaroff et al. (2001) \\
\hline \multirow[t]{2}{*}{ J1747-2809 } & $\mathrm{G} 0.9+0.1$ & as the PSR & 5.3 & Aharonian and et al. (2005) \\
\hline & & & & Porquet et al. (2003) \\
\hline J1747-2958 & G359.23-0.82 & as the PSR & 25.5 & Camilo et al. (2002b) \\
\hline J1846-0258 & Kes75 & as the PSR & 0.73 & Leahy and Tian (2008) \\
\hline $\mathrm{J} 1930+1852$ & $\mathrm{G} 54.1+0.3$ & as the PSR & 2.9 & Camilo et al. (2002a) \\
\hline $\mathrm{J} 0007+7303$ & CTA 1 & $10.2-15.8$ & 13.9 & Slane et al. (2004) \\
\hline $\mathrm{J} 0205+6449$ & $3 \mathrm{C} 58$ & $4.3-7$ & 5.4 & Slane et al. (2008) \\
\hline \multirow[t]{2}{*}{$\mathrm{J} 0538+2817$} & S147 & $40-200$ & 618.1 & Anderson et al. (1996) \\
\hline & & & & Ng et al. (2007) \\
\hline B0540-69 & 0540-693 & $0.66-1.1$ & 1.67 & Williams et al. (2008) \\
\hline B0656+14 & Monogem Ring & $86-170$ & 110.9 & Thorsett et al. (2003) \\
\hline J0821-4300 & Puppis A & $3.3-4.1$ & 1489. & Gotthelf and Halpern (2009) \\
\hline B0833-45 & Vela & $11-27$ & 11.3 & Aschenbach et al. (1995) \\
\hline J1124-5916 & $\mathrm{G} 292.0+1.8$ & $2.4-2.85$ & 2.85 & Gonzalez and Safi-Harb (2003) \\
\hline B1509-58 & G320.4-1.2 & $6-20$ & 1.6 & Yatsu et al. (2005) \\
\hline J1809-2332 & G7.5-1.7 & $10-100$ & 67.6 & Roberts and Brogan (2008) \\
\hline J1813-1749 & G12.8-0.0 & $0.285-2.5$ & 4.7 & Brogan et al. (2005) \\
\hline J1833-1034 & G21.5-0.9 & $0.8-40$. & 4.9 & Safi-Harb et al. (2001) \\
\hline
\end{tabular}


Table 1-Continued

\begin{tabular}{|c|c|c|c|c|}
\hline PSR & SNR & $\tau_{S N R} / 10^{3} \mathrm{yrs}$ & $\tau_{s d} / 10^{3} \mathrm{yrs}$ & Ref. \\
\hline B1853+01 & W44 & $6.5-20$ & 20.3 & Harrus et al. (1997) \\
\hline J1957+2831 & $\mathrm{G} 65.1+0.6$ & $40-140$ & 1568. & Tian and Leahv (2006) \\
\hline $\mathrm{B} 1951+32$ & CTB80 & $>18$ & 107. & Castelletti et al. (2003) \\
\hline B1338-62 & G308.8-0.1 & $<32.5$ & 12.1 & Caswell et al. (1992) \\
\hline J2229+6114 & G106.6+2.9 & $>3.9$ & 10.5 & Kothes et al. (2006) \\
\hline B $0531+21$ & Crab & 0.957 & 1.24 & $\underline{\text { Stephenson and Green (2002) }}$ \\
\hline J1210-5226 & G296.5+10.0 & $10-20$ & 101817. & Vasisht et al. (1997) \\
\hline J1437-5959 & G315.9-0.0 & 22 & 114. & Camilo et al. (2009) \\
\hline J1811-1925 & G11.2-0.3 & 1.6 & 23.2 & Torii et al. $(\underline{1999})$ \\
\hline $\mathrm{J} 1852+0040$ & Kes79 & 6 & 191502. & Sun et al. (2004) \\
\hline J2021+4026 & $\mathrm{G} 78.2+2.1$ & 6.6 & 76.9 & Uchivama et al. (2002) \\
\hline $\mathrm{B} 2334+61$ & G114.3+0.3 & 7.7 & 40.6 & Yar-Uvaniker et al. (2004) \\
\hline
\end{tabular}


Table 2. Spin parameters of PSRs in the sample

\begin{tabular}{|c|c|c|c|c|c|}
\hline PSR & $P \mathrm{~s}$ & $\dot{P}$ & $B / 10^{12} \mathrm{G}$ & $P_{0} \mathrm{~s}$ & $P_{0} / P$ \\
\hline J0537-6910 & 0.016 & $5.18 \mathrm{E}-14$ & 0.92 & $\ll P$ & $\sim 0$ \\
\hline J1119-6127 & 0.408 & $4.02 \mathrm{E}-12$ & 41. & $\ll P$ & $\sim 0$ \\
\hline J1747-2809 & 0.052 & $1.56 \mathrm{E}-13$ & 2.9 & $\ll P$ & $\sim 0$ \\
\hline J1747-2958 & 0.099 & $6.13 \mathrm{E}-14$ & 2.5 & $\ll P$ & $\sim 0$ \\
\hline J1846-0258 & 0.326 & $7.08 \mathrm{E}-12$ & 48.6 & $\ll P$ & $\sim 0$ \\
\hline $\mathrm{J} 1930+1852$ & 0.137 & $7.51 \mathrm{E}-13$ & 10.3 & $\ll P$ & $\sim 0$ \\
\hline $\mathrm{J} 0007+7303$ & 0.316 & $3.6 \mathrm{E}-13$ & 10.8 & $<0.163$ & $<0.52$ \\
\hline $\mathrm{J} 0205+6449$ & 0.066 & $1.94 \mathrm{E}-13$ & 3.6 & $<0.029$ & $<0.45$ \\
\hline \multirow[t]{2}{*}{ J0538+2817 } & 0.143 & $3.67 \mathrm{E}-15$ & 0.73 & $<0.134$ & $<0.93$ \\
\hline & 0.143 & $3.67 \mathrm{E}-15$ & 0.73 & $>0.118$ & $>0.82$ \\
\hline \multirow[t]{2}{*}{ B0540-69 } & 0.05 & $4.79 \mathrm{E}-13$ & 5.0 & $<0.039$ & $<0.78$ \\
\hline & 0.05 & $4.79 \mathrm{E}-13$ & 5.0 & $>0.03$ & $>0.59$ \\
\hline B0656+14 & 0.385 & $5.5 \mathrm{E}-14$ & 4.7 & $<0.183$ & $<0.48$ \\
\hline \multirow[t]{2}{*}{ J0821-4300 } & 0.113 & $1.2 \mathrm{E}-15$ & 0.37 & $<0.113$ & $\sim 1$ \\
\hline & 0.113 & $1.2 \mathrm{E}-15$ & 0.37 & $>0.113$ & $\sim 1$ \\
\hline B0833-45 & 0.089 & $1.25 \mathrm{E}-13$ & 3.4 & $<0.016$ & $<0.2$ \\
\hline \multirow[t]{2}{*}{ J1124-5916 } & 0.135 & $7.53 \mathrm{E}-13$ & 10.2 & $<0.054$ & $<0.40$ \\
\hline & 0.135 & $7.53 \mathrm{E}-13$ & 10.2 & $>0.004$ & $>0.03$ \\
\hline J1210-5226 & 0.424 & $6.6 \mathrm{E}-17$ & 0.17 & 0.424 & $\sim 1$ \\
\hline B1509-58 & 0.151 & $1.54 \mathrm{E}-12$ & 15.4 & - & - \\
\hline
\end{tabular}


Table 2-Continued

\begin{tabular}{|c|c|c|c|c|c|}
\hline PSR & $P \mathrm{~s}$ & $\dot{P}$ & $B / 10^{12} \mathrm{G}$ & $P_{0} \mathrm{~s}$ & $P_{0} / P$ \\
\hline J1809-2332 & 0.147 & $3.44 \mathrm{E}-14$ & 2.3 & $<0.136$ & $<0.92$ \\
\hline \multirow[t]{2}{*}{ J1813-1749 } & 0.045 & $1.5 \mathrm{E}-13$ & 2.6 & $<0.043$ & $<0.97$ \\
\hline & 0.045 & $1.5 \mathrm{E}-13$ & 2.6 & $>0.031$ & $>0.69$ \\
\hline J1833-1034 & 0.062 & $2.02 \mathrm{E}-13$ & 3.6 & $<0.057$ & $<0.91$ \\
\hline \multirow[t]{2}{*}{ B1853+01 } & 0.267 & $2.08 \mathrm{E}-13$ & 7.5 & $<0.221$ & $<0.83$ \\
\hline & 0.267 & $2.08 \mathrm{E}-13$ & 7.5 & $>0.036$ & $>0.14$ \\
\hline \multirow[t]{2}{*}{ J1957+2831 } & 0.308 & $3.11 \mathrm{E}-15$ & 0.99 & $<0.3$ & $<0.99$ \\
\hline & 0.308 & $3.11 \mathrm{E}-15$ & 0.99 & $>0.29$ & $>0.95$ \\
\hline B1951+32 & 0.04 & $5.84 \mathrm{E}-15$ & 0.49 & $<0.036$ & $<0.91$ \\
\hline B1338-62 & 0.193 & $2.53 \mathrm{E}-13$ & 7.1 & - & - \\
\hline J2229+6114 & 0.052 & $7.83 \mathrm{E}-14$ & 2.0 & $<0.041$ & $<0.79$ \\
\hline B $0531+21$ & 0.033 & $4.23 \mathrm{E}-13$ & 3.8 & 0.016 & 0.48 \\
\hline J1437-5959 & 0.062 & $8.59 \mathrm{E}-15$ & 0.74 & 0.055 & 0.9 \\
\hline J1811-1925 & 0.065 & 4.40E-14 & 1.7 & 0.062 & 0.97 \\
\hline $\mathrm{J} 1852+0040$ & 0.105 & $8.68 \mathrm{E}-18$ & 0.03 & 0.105 & $\sim 1$ \\
\hline $\mathrm{J} 2021+4026$ & 0.265 & $5.47 \mathrm{E}-14$ & 3.9 & 0.254 & 0.96 \\
\hline B2334+61 & 0.495 & $1.93 \mathrm{E}-13$ & 9.9 & 0.45 & 0.91 \\
\hline
\end{tabular}




\section{Discussion}

For our estimates we use a braking index $n=3$. Additional checks were made for other values, $2<n<10$, and we found that the derived values of $P_{0}$ are not significantly changed in many cases. For $n=2 P_{0}$ may be larger by a factor up to 2 , but these changes do not modify the general picture shown in Fig. 1 significantly. This is an expected result, since the objects under consideration are very young, and is in correspondence with the

conclusions by Faucher-Giguère and Kaspi (2006) that varying $n$ around the standard value has a weak impact on the parameters of the pulsar population. However, with growing $n$ more and more SNR ages are found to be in contradiction with the spin-down ages. Already for $n=4$ in several cases $\tau_{\text {sd }}$ become smaller than $\tau_{\mathrm{SNR}}$.

Recently, an e-print by Zhang and Xie (2011) appeared in which a correlation between magnetic field and the ratio of the "true" (i.e. $\tau_{\mathrm{SNR}}$ ) and spin-down ages was proposed. The authors explain this correlation as due to field decay. We present a similar plot, i.e. $B$ vs. ages ratio, in Fig. 2 using data from Table 2. However, we believe that the existence of such a correlation is an artifact, and the reason for its appearance is that Zhang and Xie (2011) neglect the initial spin period. This conclusion can be illustrated by plotting $B$ vs. the difference $P-P_{0}$. Clearly, in the sample of young objects (associated with SNRs) those with smaller magnetic fields have initial spin periods closer to the present day periods.

Naturally, objects with lower field, like the "anti-magnetars" associated to some central compact objects in SNRs, have present spin periods very close to the initial values. Then, the usual estimate of the spin-down age assuming $P_{0} \simeq 0$ is invalid, as the true age is very different.

We cannot exclude that there was some field evolution in the objects under discussion (note that field evolution is undistinguishable from the evolution of the angle between spin and magnetic axis), but we see no evidence for it, contrary to the conclusion by 


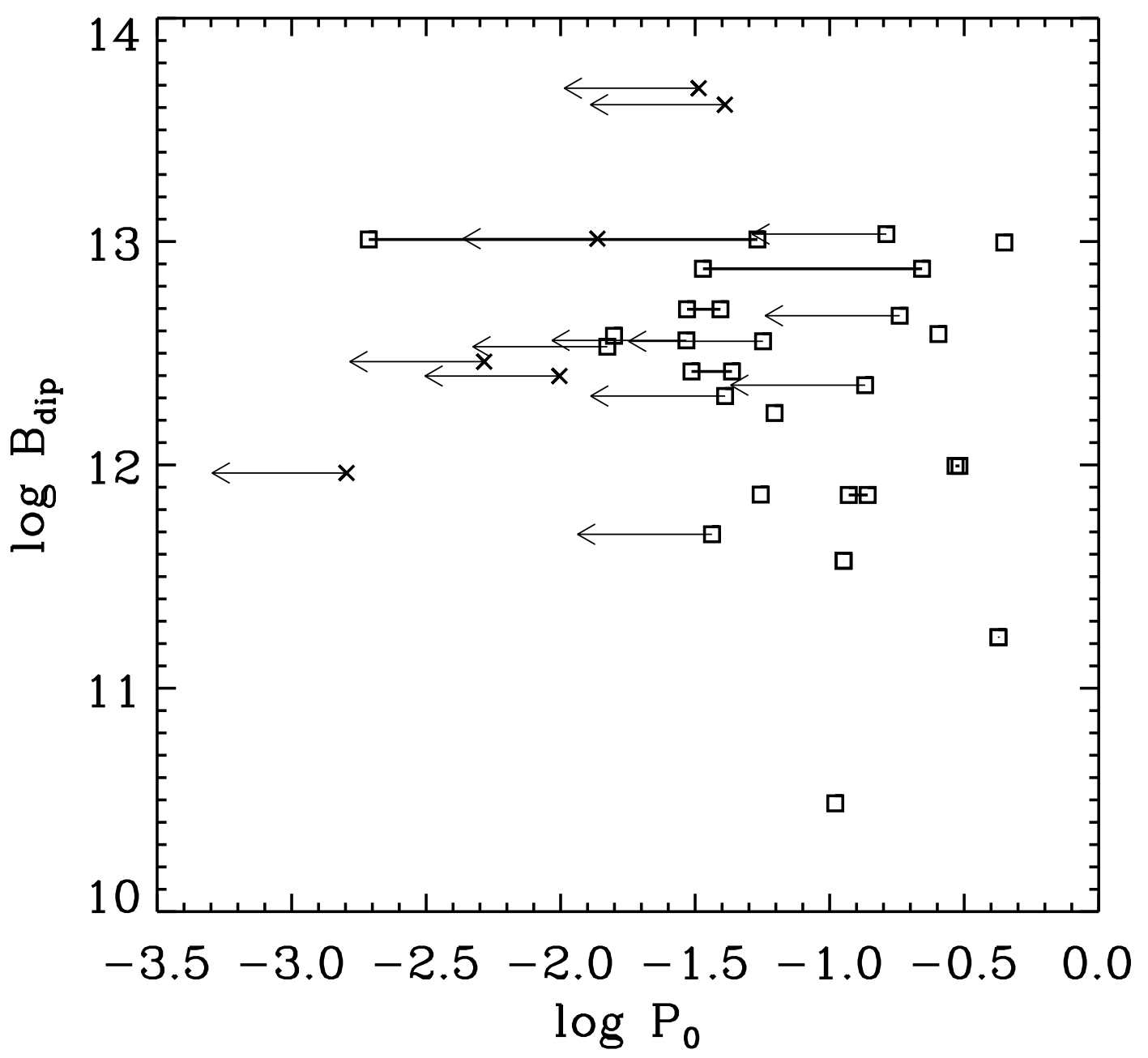

Fig. 1. - Magnetic fields and initial spin periods for 22 PSRs (squares). In several cases only upper limits are available (shown with arrows). For the group of six pulsars with SNR ages in correspondence with spin-down ages we use different symbols (crosses) and assume that $P_{0}<0.1 P$, where $P$ is the present period. All values are estimated for standard magnetodipole losses in the case of constant field and angle between spin and magnetic axis. 


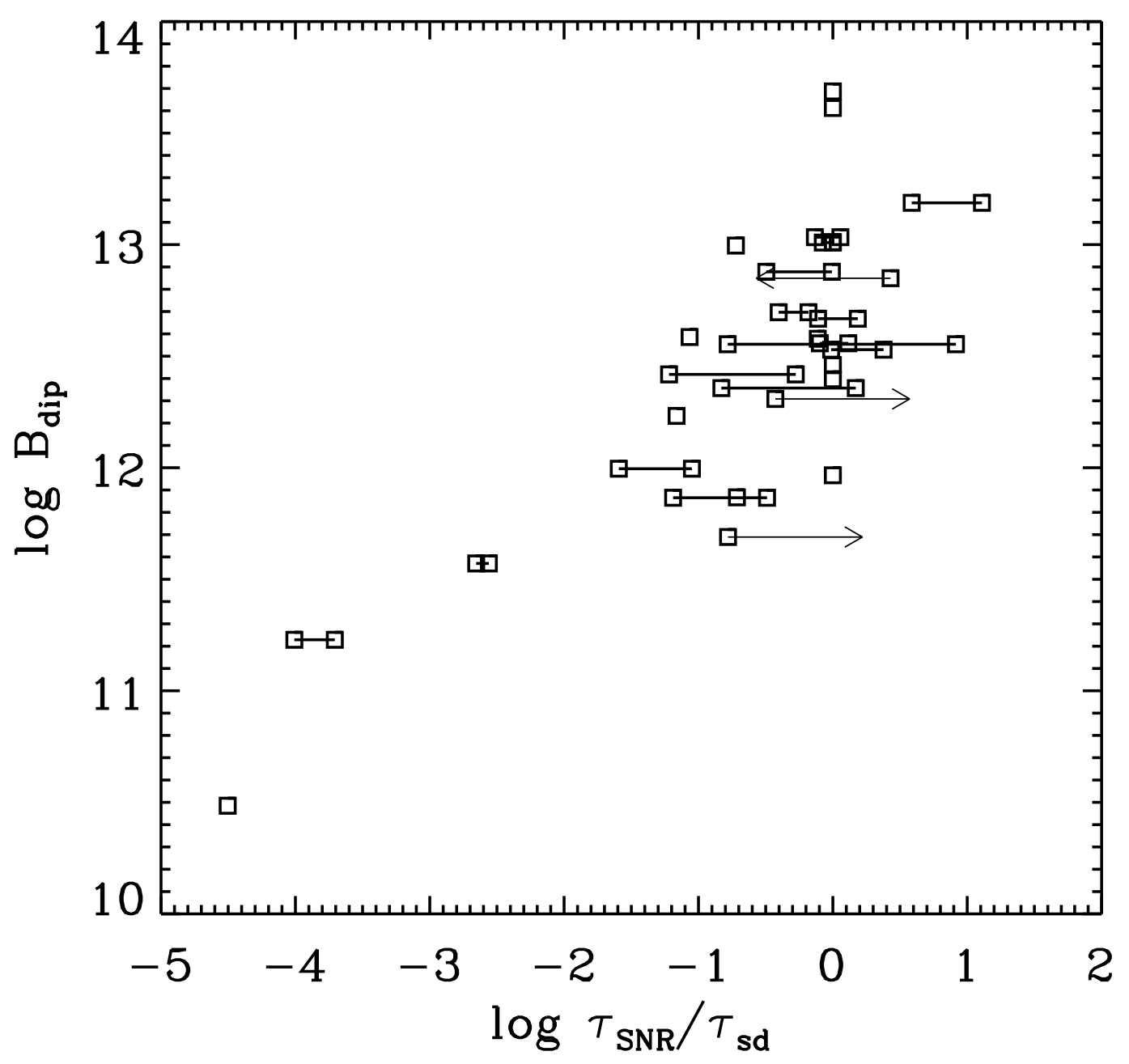

Fig. 2.- A plot similar to the one in Zhang and Xie (2011): magnetic field vs. the ratio of "true" age $\left(\tau_{\mathrm{SNR}}\right)$ and spin-down age. 
Zhang and Xie (2011).

Not surprisingly, the assumptions made by Zhang and Xie (2011) led them to suggest (Zhang and Xie 2012) that field decay is so strong and important that in their youth most of standard pulsars have magnetar properties, $B \sim 510^{14} \mathrm{G}$ at the age $\sim 0.4 \mathrm{yrs}$. We believe that this is not needed if non-zero initial spin periods are accounted for.

Oscillations of braking indices were also proposed by Biryukov et al. (2012) on a time scale $\sim 10^{3}-10^{4}$ yrs. If this is valid also for young pulsars, then our estimates of $P_{0}$ must be corrected. But still, non-zero $P_{0}$ will remain the main reason to explain discrepancy between spin-down ages and SNR ages.

In Fig. 1 we see that under the assumptions we made no correlation between $P_{0}$ and $B$ is visible. This is in correspondence with standard assumptions made in different kinds of models. We did not try to figure out a possible shape of the initial spin period distribution (for example, if it is a multi-peak distribution or not — in the future, probably, it will appear that, for example, so-called "anti-magnetars" form a separate subpopulation, and so they do not fit the same single-mode distribution as normal radio pulsars; if gaussian is better than log-gaussian, symmetric distributions better than asymmetric, etc.). However, the data we obtained can be used to check assumptions about initial spin distributions. For example, obviously very narrow distributions are not in correspondence with the data as there are pulsars with $P_{0}$ from milliseconds to hundreds of milliseconds.

In order to derive information on the distribution of the initial periods from the values derived from observations, we run a number of Kolmogorov-Smirnov (KS) tests, using different forms of the distribution function. In particular, we tested gaussian distributions with different parameters and a top-hat (i.e. constant in a range and zero outside) distribution. For this calculations we used data on 11 PSRs. Six of the are those with an exactly determined $P_{0}$, to which we added five objects with small uncertainty for the initial 
period value using average SNR age.

Two representative examples are shown in Figs. 3, 4. The gaussian distribution of Fig. 3 gives a KS significance level of $\sim 0.66$, indicating that the data and model have quite consistent cumulative distributions. On the other hand, the top-hat distribution has a very low value of the KS significance, $<0.01$, and should be rejected.

\section{Conclusions}

In this paper we discussed a sample of radio pulsars associated with supernova remnants. This association allows to obtain estimates of initial spin periods in $\gtrsim 20$ cases. The obtained distribution is rather flat in wide range from $\sim 10 \mathrm{msec}$ to hundreds of msec.

We thank Andrea Possenti for some helpful comments on the paper. SP also thanks Anton Biryukov and Andrei Igoshev for discussions. The work of S.P. was supported by RFBR grants (10-02-00599, 12-02-00186) and by the Federal programm for scientific and educational staff (02.740.11.0575). RT acknowledges financial support by INAF through a PRIN 2011 grant. SP thanks the University of Padova for hospitality. 


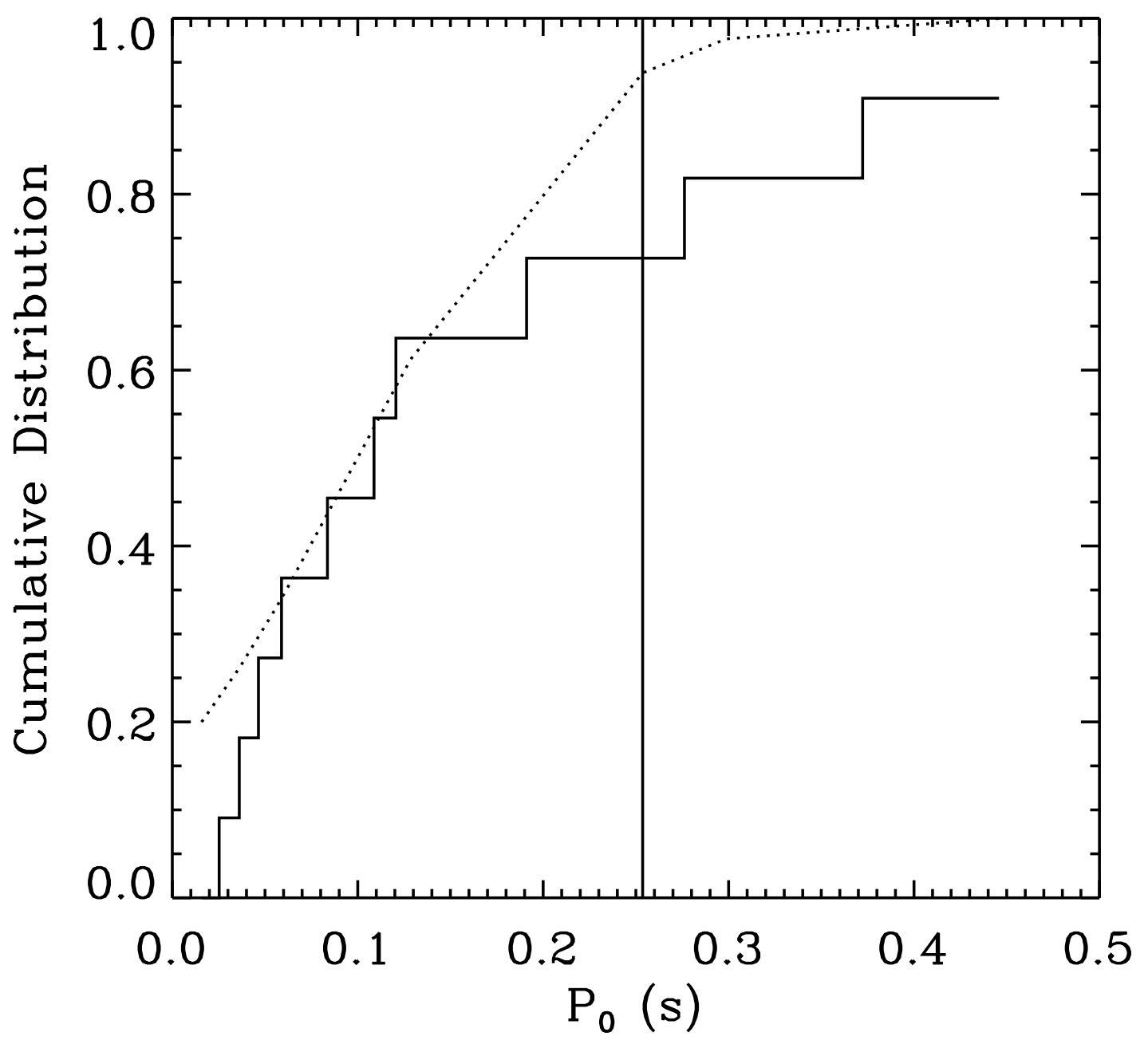

Fig. 3.- The data cumulative distribution function (full histogram) compared with that of a gaussian distribution with average $0.1 \mathrm{~s}$ and standard deviation of $0.1 \mathrm{~s}$ (dotted line). The vertical line shows the value of $P_{0}$ where the difference is largest. 


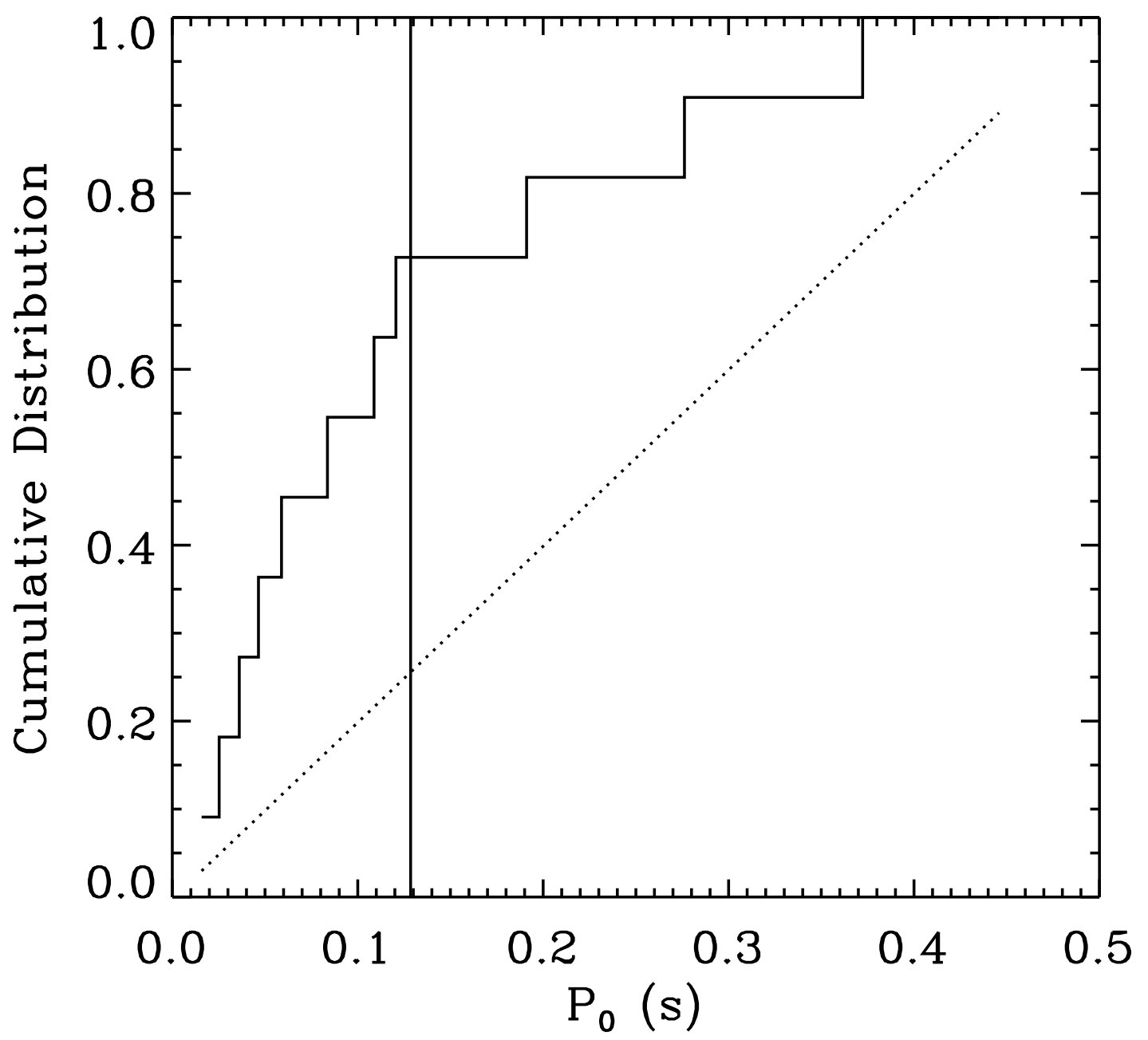

Fig. 4.- Same as in Fig. 3 for a top-hat distribution in the range $0.001 \mathrm{~s} \leq P_{0} \leq 0.5 \mathrm{~s}$. 


\section{REFERENCES}

Abdo, A.A., et al.: Science 327, 1103 (2010). doi:10.1126/science.1182787

Aharonian, F., et al.: A\&A 432, 25 (2005). arXiv:astro-ph/0501265. doi:10.1051/00046361:200500022

Anderson, S.B., Cadwell, B.J., Jacoby, B.A., Wolszczan, A., Foster, R.S., Kramer, M.: ApJ 468, 55 (1996). doi:10.1086/310218

Aschenbach, B., Egger, R., Trümper, J.: Nature 373, 587 (1995). doi:10.1038/373587a0

Bietenholz, M.F.: ApJ 645, 1180 (2006). arXiv:astro-ph/0603197. doi:10.1086/504584

Biryukov, A., Beskin, G., Karpov, S.: MNRAS 420, 103 (2012). 1105.5019. doi:10.1111/j.1365-2966.2011.20005.x

Brogan, C.L., Gaensler, B.M., Gelfand, J.D., Lazendic, J.S., Lazio, T.J.W., Kassim, N.E., McClure-Griffiths, N.M.: ApJ 629, 105 (2005). arXiv:astro-ph/0505145. doi:10.1086/491471

Camilo, F., Lorimer, D.R., Bhat, N.D.R., Gotthelf, E.V., Halpern, J.P., Wang, Q.D., Lu, F.J., Mirabal, N.: ApJ 574, 71 (2002a). arXiv:astro-ph/0206220. doi:10.1086/342351

Camilo, F., Manchester, R.N., Gaensler, B.M., Lorimer, D.R.: ApJ 579, 25 (2002b). arXiv:astro-ph/0209480. doi:10.1086/344832

Camilo, F., Ng, C.-Y., Gaensler, B.M., Ransom, S.M., Chatterjee, S., Reynolds, J., Sarkissian, J.: ApJ 703, 55 (2009). 0908.2421. doi:10.1088/0004-637X/703/1/L55

Castelletti, G., Dubner, G., Golap, K., Goss, W.M., Velázquez, P.F., Holdaway, M., Rao, A.P.: AJ 126, 2114 (2003). arXiv:astro-ph/0310655. doi:10.1086/378482 
Caswell, J.L., Kesteven, M.J., Stewart, R.T., Milne, D.K., Haynes, R.F.: ApJ 399, 151 (1992). doi:10.1086/186629

Chevalier, R.A.: ApJ 619, 839 (2005). arXiv:astro-ph/0409013. doi:10.1086/426584

Dean, A.J., Hill, A.B.: A\&A 485, 195 (2008). 0804.3420. doi:10.1051/0004-6361:200809356

Faucher-Giguère, C.-A., Kaspi, V.M.: ApJ 643, 332 (2006). arXiv:astro-ph/0512585. doi:10.1086/501516

Fryer, C.L., Belczynski, K., Wiktorowicz, G., Dominik, M., Kalogera, V., Holz, D.: ArXiv e-prints (2011). 1110.1726

Gonzalez, M., Safi-Harb, S.: ApJ 583, 91 (2003). arXiv:astro-ph/0301193. doi: $10.1086 / 368122$

Gotthelf, E.V., Halpern, J.P.: ApJ 695, 35 (2009). 0902.3007. doi:10.1088/0004$637 X / 695 / 1 /$ L35

Green, D.A., Stephenson, F.R.: In: K. Weiler (ed.) Supernovae and Gamma-Ray Bursters. Lecture Notes in Physics, Berlin Springer Verlag, vol. 598, p. 7 (2003). arXiv:astro-ph/0301603

Haensel, P., Potekhin, A.Y., Yakovlev, D.G. (eds.): Neutron Stars 1 : Equation of State and Structure. Astrophysics and Space Science Library, vol. 326 (2007)

Halpern, J.P., Gotthelf, E.V., Camilo, F., Helfand, D.J., Ransom, S.M.: ApJ 612, 398 (2004). arXiv:astro-ph/0404312. doi:10.1086/422409

Harrus, I.M., Hughes, J.P., Singh, K.P., Koyama, K., Asaoka, I.: ApJ 488, 781 (1997). arXiv:astro-ph/9705239. doi:10.1086/304717 
Kellett, B.J., Branduardi-Raymont, G., Culhane, J.L., Mason, I.M., Mason, K.O., Whitehouse, D.R.: MNRAS 225, 199 (1987)

Kirshner, R.P., Morse, J.A., Winkler, P.F., Blair, W.P.: ApJ 342, 260 (1989). doi: $10.1086 / 167590$

Kothes, R., Reich, W., Uyanıker, B.: ApJ 638, 225 (2006). doi:10.1086/498666

Kundu, M.R., Angerhofer, P.E., Fuerst, E., Hirth, W.: A\&A 92, 225 (1980)

Lattimer, J.M., Prakash, M.: Science 304, 536 (2004). arXiv:astro-ph/0405262. doi:10.1126/science. 1090720

Leahy, D.A., Tian, W.W.: A\&A 480, 25 (2008). 0711.4107. doi:10.1051/0004-6361:20079149

Manchester, R.N., Staveley-Smith, L., Kesteven, M.J.: ApJ 411, 756 (1993). doi:10.1086/172877

Manchester, R.N., Hobbs, G.B., Teoh, A., Hobbs, M.: AJ 129, 1993 (2005). doi: $10.1086 / 428488$

Marshall, F.E., Gotthelf, E.V., Zhang, W., Middleditch, J., Wang, Q.D.: ApJ 499, 179 (1998). arXiv:astro-ph/9803214. doi:10.1086/311381

Migliazzo, J.M., Gaensler, B.M., Backer, D.C., Stappers, B.W., van der Swaluw, E., Strom, R.G.: ApJ 567, 141 (2002). arXiv:astro-ph/0202063. doi:10.1086/340002

Ng, C.-Y., Romani, R.W., Brisken, W.F., Chatterjee, S., Kramer, M.: ApJ 654, 487 (2007). arXiv:astro-ph/0611068. doi:10.1086/510576

Park, S., Hughes, J.P., Slane, P.O., Mori, K., Burrows, D.N.: ApJ 710, 948 (2010). 0912.5177. doi:10.1088/0004-637X/710/2/948 
Pivovaroff, M.J., Kaspi, V.M., Camilo, F., Gaensler, B.M., Crawford, F.: ApJ 554, 161 (2001). arXiv:astro-ph/0102084. doi:10.1086/321340

Plucinsky, P.P., Snowden, S.L., Aschenbach, B., Egger, R., Edgar, R.J., McCammon, D.: ApJ 463, 224 (1996). doi:10.1086/177236

Popov, S.B., Prokhorov, M.E.: Physics Uspekhi 50, 1123 (2007). arXiv:astro-ph/0411792. doi:10.1070/PU2007v050n11ABEH006179

Popov, S.B., Pons, J.A., Miralles, J.A., Boldin, P.A., Posselt, B.: MNRAS 401, 2675 (2010). 0910.2190. doi:10.1111/j.1365-2966.2009.15850.x

Porquet, D., Decourchelle, A., Warwick, R.S.: A\&A 401, 197 (2003). arXiv:astro-ph/0211426. doi:10.1051/0004-6361:20021670

Reynolds, S.P.: ApJ 291, 152 (1985). doi:10.1086/163050

Roberts, M.S.E., Brogan, C.L.: ApJ 681, 320 (2008). 0802.3750. doi:10.1086/588419

Rudie, G.C., Fesen, R.A.: In: Revista Mexicana de Astronomia y Astrofisica Conference Series. Revista Mexicana de Astronomia y Astrofisica, vol. 27, vol. 30, p. 90 (2007). 0704.2780

Safi-Harb, S., Harrus, I.M., Petre, R., Pavlov, G.G., Koptsevich, A.B., Sanwal, D.: ApJ 561, 308 (2001). arXiv:astro-ph/0107175. doi:10.1086/322978

Scheck, L., Kifonidis, K., Janka, H.-T., Müller, E.: A\&A 457, 963 (2006). arXiv:astroph/0601302. doi:10.1051/0004-6361:20064855

Slane, P., Zimmerman, E.R., Hughes, J.P., Seward, F.D., Gaensler, B.M., Clarke, M.J.: ApJ 601, 1045 (2004). arXiv:astro-ph/0310250. doi:10.1086/380498 
Slane, P., Helfand, D.J., Reynolds, S.P., Gaensler, B.M., Lemiere, A., Wang, Z.: ApJ 676, 33 (2008). 0802.0206. doi:10.1086/587031

Stephenson, F.R., Green, D.A.: Historical supernovae and their remnants, by F. Richard Stephenson and David A. Green. International series in astronomy and astrophysics, vol. 5. Oxford: Clarendon Press, 2002, ISBN 01985076665 (2002)

Sun, M., Seward, F.D., Smith, R.K., Slane, P.O.: ApJ 605, 742 (2004). arXiv:astroph/0401165. doi:10.1086/382666

Thorsett, S.E., Benjamin, R.A., Brisken, W.F., Golden, A., Goss, W.M.: ApJ 592, 71 (2003). arXiv:astro-ph/0306462. doi:10.1086/377682

Tian, W.W., Leahy, D.A.: A\&A 455, 1053 (2006). arXiv:astro-ph/0603102. doi:10.1051/00046361:20065140

Torii, K., Tsunemi, H., Dotani, T., Mitsuda, K., Kawai, N., Kinugasa, K., Saito, Y., Shibata, S.: ApJ 523, 69 (1999). doi:10.1086/312251

Uchiyama, Y., Takahashi, T., Aharonian, F.A., Mattox, J.R.: ApJ 571, 866 (2002). arXiv:astro-ph/0202414. doi:10.1086/340121

Vasisht, G., Aoki, T., Dotani, T., Kulkarni, S.R., Nagase, F.: ApJ 456, 59 (1996). doi:10.1086/309854

Vasisht, G., Kulkarni, S.R., Anderson, S.B., Hamilton, T.T., Kawai, N.: ApJ 476, 43 (1997). doi:10.1086/310493

Wang, Q.D., Gotthelf, E.V.: ApJ 494, 623 (1998). arXiv:astro-ph/9708087. doi:10.1086/305214 
Williams, B.J., Borkowski, K.J., Reynolds, S.P., Raymond, J.C., Long, K.S., Morse, J., Blair, W.P., Ghavamian, P., Sankrit, R., Hendrick, S.P., Smith, R.C., Points, S., Winkler, P.F.: ApJ 687, 1054 (2008). 0807.4155. doi:10.1086/592139

Winkler, P.F., Tuttle, J.H., Kirshner, R.P., Irwin, M.J.: In: R. S. Roger \& T. L. Landecker (ed.) IAU Colloq. 101: Supernova Remnants and the Interstellar Medium, p. 65 (1988)

Yar-Uyaniker, A., Uyaniker, B., Kothes, R.: ApJ 616, 247 (2004). arXiv:astro-ph/0408386. doi:10.1086/424794

Yatsu, Y., Kataoka, J., Kawai, N., Tamura, K., Brinkmann, W.: Advances in Space Research 35, 1066 (2005). doi:10.1016/j.asr.2005.05.015

Zhang, S.-N., Xie, Y.: ArXiv e-prints (2011). 1110.3154

Zhang, S.-N., Xie, Y.: ArXiv e-prints (2012). 1202.1123 